\title{
THE DIMENSION OF THE FIXED POINT SET ON AFFINE FLAG MANIFOLDS
}

\author{
ROMAN BEZRUKAVNIKOV
}

Let $G$ be a semisimple simply-connected algebraic group over $\mathbb{C}, \mathfrak{g}$ its Lie algebra. Also, $F=\mathbb{C}((\varepsilon))$ is the field of formal Laurent series, $A=\mathbb{C}[[\varepsilon]]$ is the ring of integers in $F$. Set $\hat{\mathfrak{g}}=\mathfrak{g} \otimes F, \mathfrak{g}_{A}=\mathfrak{g} \otimes A$ and $\hat{G}=G(F)$.

Let $\mathcal{B}$ be the set of all Iwahori subalgebras in $\hat{\mathfrak{g}}$, and $X$ the set of all subalgebras in $\hat{\mathfrak{g}}$ which are $\hat{G}$-conjugate to $\mathfrak{g}_{A}$. Then $\mathcal{B}$ and $X$ have the structure of Ind-algebraic varieties over $\mathbb{C}$ (they are unions of increasing system of ordinary projective algebraic varieties over $\mathbb{C}$ ). They are called the affine flag variety and the affine Grassmanian of $G$ respectively. We have $X=\hat{G} / G(A)$ and $\mathcal{B}=\hat{G} / I$, where $I$ is an Iwahori subgroup.

For any $N \in \hat{\mathfrak{g}}$ let $\mathcal{B}_{N} \subset \mathcal{B}$ (respectively $X_{N} \subset X$ ) be the set of all Iwahori subalgebras (respectively, subalgebras conjugate to $\mathfrak{g}_{A}$ ) which contain $N$. Clearly, $\mathcal{B}_{N}\left(X_{N}\right)$ is a closed subvariety of the Ind-variety $\mathcal{B}$ (respectively $X$ ).

The varieties $\mathcal{B}_{N}, X_{N}$ were studied by Kazhdan and Lusztig in [KL]. Following their paper let us suppose that $N$ is topologically nilpotent (nilelement in the terminology of $[\mathrm{KL}])$, i.e., $\operatorname{ad}(N)^{r} \rightarrow 0$ in $\operatorname{End}_{F}(\hat{\mathfrak{g}})$ when $r \rightarrow \infty$. (The topology on $\operatorname{End}_{F}(\hat{\mathfrak{g}})$ arises from the obvious topology on $F$.) It was shown in loc. cit. that the Ind-varieties $\mathcal{B}_{N}, X_{N}$ are finite dimensional iff the element $N$ is regular semisimple. We will assume from now on that this is the case. Then $\mathcal{B}_{N}$ and $X_{N}$ are locally finite unions of finite dimensional projective varieties. Moreover, all components of $\mathcal{B}_{N}$ have the same dimension, which coincides with the dimension of $X_{N}$. A precise formula for the dimension of $\mathcal{B}_{N}$ was stated in $[\mathrm{KL}]$ as a conjecture. The aim of the present note is to give a proof of this conjecture.

Let $O$ be the subset of $X_{N}$ defined as follows: if $\hat{\mathfrak{p}} \in X_{N}$ is a subalgebra conjugate to $\mathfrak{g}_{A}$, then $\hat{\mathfrak{p}} \in O$ iff the image of $N$ in $\mathfrak{g} \cong \hat{\mathfrak{p}} / \varepsilon \hat{\mathfrak{p}}$ is a regular nilpotent.

Let $Z(N)$ be the centralizer of $N$ in $\hat{G}$; let $\mathfrak{z}(N)$ be the centralizer of $N$ in $\hat{\mathfrak{g}}$. We also fix a Cartan subalgebra $\mathfrak{h} \subset \mathfrak{g}$ and denote by $W$ the Weyl group. The result containing the formula for the dimension of $\mathcal{B}_{N}$ is the following:

Received December 3, 1995. 


\section{Proposition.}

a) $O$ is an orbit of the group $Z(N)$.

b) The dimension of this orbit is given by the formula (cf. [KL], p.130)

$$
\operatorname{dim}(O)=1 / 2\left(\delta(N)-\operatorname{rk}(\mathfrak{g})+\operatorname{dim}\left(\mathfrak{h}^{w}\right)\right)
$$

where $w \in W$ is such that $\mathfrak{z}(N)$ is of type $w$ (see [KL], $\S 1$ ); $\mathfrak{h}^{w}$ denotes the $w$-invariants in $\mathfrak{h}$ and $\delta(N)$ is the valuation of

$$
\operatorname{det}(\operatorname{ad} N: \hat{\mathfrak{g}} / \mathfrak{z}(N) \longrightarrow \hat{\mathfrak{g}} / \mathfrak{z}(N)) .
$$

c) $\operatorname{dim}\left(\mathcal{B}_{N}\right)=\operatorname{dim}\left(X_{N}\right)=\operatorname{dim}(O)$.

Proof. a) is equivalent to the following:

Claim. Suppose that $N, N^{\prime}$ are nil-elements in $\mathfrak{g}_{A}$, which lie in the same $\hat{G}$ orbit, and the images of $N$ and $N^{\prime}$ in $\mathfrak{g}=\mathfrak{g}_{A} / \varepsilon \mathfrak{g}_{A}$ are regular nilpotents. Then $N$ and $N^{\prime}$ lie in one $G(A)$ orbit.

Proof of the claim. Since the regular nilpotents in $\mathfrak{g}$ form one conjugacy class we can assume that $N \bmod \varepsilon \mathfrak{g}_{A}=N^{\prime} \bmod \varepsilon \mathfrak{g}_{A}=n$, where $n \in \mathfrak{g}$ is a regular nilpotent.

Let $V$ be any complement to the image of $\operatorname{ad}(n)$ in $\mathfrak{g}$, and let $v_{1}, \ldots, v_{r}$ be a basis of $V$.

\section{Lemma 1.}

a) Assume that $x \in \mathfrak{g}_{A}$ is such that $x \in n+\varepsilon V \otimes A+\varepsilon^{i} \mathfrak{g}_{A}$ for some $i \geq 1$. Then there exists $g \in G(A)$ such that $g=1 \bmod \varepsilon^{i}$ and $\operatorname{ad}(g)(x) \in n+\varepsilon V \otimes A+\varepsilon^{i+1} \mathfrak{g}_{A}$.

b) Any such $x$ is $G(A)$-conjugate to an element lying in $n+\varepsilon V \otimes A$.

Proof. To prove a) let us write $x$ as $x=n+v+\left[n, \varepsilon^{i} y\right]$ for some $v \in \varepsilon V \otimes A$, $y \in \mathfrak{g}_{A}$. (This is possible because $\varepsilon^{i} \mathfrak{g}_{A} \subset \varepsilon V \otimes A+\operatorname{ad}(n)\left(\varepsilon^{i} \mathfrak{g}_{A}\right)$ as follows from the definition of $V)$. It is enough to take $g=\exp \left(-\varepsilon^{i} y\right)$.

To prove b) note that by the statement a) there exists a sequence of elements $g_{i} \in G(A)$ such that $g_{i}=g_{i+1} \bmod \varepsilon^{i}$ and $\operatorname{ad}\left(g_{i}\right)(x) \in n+\varepsilon V \otimes$ $A+\varepsilon^{i} \mathfrak{g}_{A}$. It is obvious that $g:=\lim g_{i}$ exists, lies in $G(A)$ and satisfies

$$
\operatorname{ad}(g)(x) \in n+\varepsilon V \otimes A .
$$

Now we see that $N$ (respectively $N^{\prime}$ ) is $G(A)$-conjugate to an element of the form $N_{0}=n+\Sigma a_{i} v_{i}$ (respectively $N_{0}^{\prime}=n+\Sigma a_{i}^{\prime} v_{i}$ ), where $a_{i}, a_{i}^{\prime} \in \varepsilon A$. By the theorem of Kostant (see [K], Theorem 0.10) there exists a set of generators $Q_{1}, . ., Q_{r}$ of the ring of invariant polynomials on $\mathfrak{g}$ such that

$$
Q_{k}\left(n+\Sigma a_{i} v_{i}\right)=a_{k}
$$


Since $N^{\prime}, N_{0}^{\prime}, N, N_{0}$ lie in one $\hat{G}$ orbit, we have $Q_{i}\left(N_{0}\right)=Q_{i}\left(N_{0}^{\prime}\right)$, hence $a_{i}=a_{i}^{\prime}$ and $N_{0}=N_{0}^{\prime}$. The claim is proved.

c) It is clear that $O$ is open; $O$ is nonempty by [KL], $\S 4$, Corollary 1 . The natural projection $\pi: \mathcal{B}_{N} \longrightarrow X_{N}$ is $1-1$ over $O$. Since $\pi^{-1}(O)$ is open in $\mathcal{B}_{N}$ and all components of $\mathcal{B}_{N}$ have the same dimension ([KL], $\S 4$, Proposition 1) we see that $\operatorname{dim}(O)=\operatorname{dim}\left(\pi^{-1}(O)\right)=\operatorname{dim}\left(\mathcal{B}_{N}\right)=$ $\operatorname{dim}\left(X_{N}\right)$. (The last equality is Corollary $2, \S 4$ of $[\mathrm{KL}]$.)

b) For split $N$ the formula for the dimension of $X_{N}$ follows from [KL], $\S 5$ (and coincides with the formula b) above) so by the statement (c) we are done. The general case can be reduced to the case of split $N$ by the next two lemmas.

Let $N \in \hat{\mathfrak{g}}$ be any regular semisimple nil-element. Consider the field extension $\widetilde{F} / F$ of degree $n$, and the corresponding ring extension $\widetilde{A} / A$, such that $N$ splits over $\widetilde{F}$. We have $\widetilde{F} \cong \mathbb{C}\left(\left(\varepsilon^{1 / n}\right)\right) ; \widetilde{A} \cong \mathbb{C}\left[\left[\varepsilon^{1 / n}\right]\right]$. Let $\widetilde{X}=G(\widetilde{F}) / G(\widetilde{A})$ be the corresponding affine Grassmanian, and $\widetilde{Z}(N)$ be the centralizer of $N$ in $G(\widetilde{F})$.

For any $\hat{\mathfrak{p}} \in X$ consider the orbit of $Z(N)$ on $X$ containing $\hat{\mathfrak{p}}$, and the orbit of $\widetilde{Z}(N)$ on $\widetilde{X}$ containing $\hat{\mathfrak{p}} \otimes \widetilde{A} \in \widetilde{X}$. They will be denoted by $O_{\hat{\mathfrak{p}}}$ and $\widetilde{O}_{\hat{\mathfrak{p}}}$ respectively. Also let $\hat{P} \subset \hat{G}$ (respectively $\widetilde{P} \subset G(\widetilde{F})$ ) denote the stabilizer of $\hat{\mathfrak{p}}$ (respectively the stabilizer of $\hat{\mathfrak{p}} \otimes \widetilde{A}$ ).

Let us call an element $x \in \hat{\mathfrak{g}}$ (respectively $y \in \mathfrak{g} \otimes \widetilde{F}$ ) integral if for any ad-invariant polynomial $Q$ which is defined over $\mathbb{C}$ we have $Q(x) \in A$ (respectively $Q(y) \in \widetilde{A}$ ). It is easy to see that the integral elements in $\mathfrak{z}(N)$ form a lattice, provided $N$ is regular semisimple. The exponent is a surjective homomorphism with discrete kernel from this lattice to the connected component of $Z(N)$.

Let $M$ (respectively $\widetilde{M}$ ) be the lattice of integral elements in $\mathfrak{z}(N)$ (respectively in $\mathfrak{z}(N) \otimes \widetilde{F})$. The Killing form will be denoted by $k$.

Lemma 2. For any $\hat{\mathfrak{p}} \in X$ we have

$$
\operatorname{dim}\left(\widetilde{O}_{\hat{\mathfrak{p}}}\right)=n\left[\operatorname{dim}\left(O_{\hat{\mathfrak{p}}}\right)+1 / 2 v(\operatorname{det}(k \mid M))\right]
$$

where $v_{F}$ is the valuation of $F$.

Proof. Indeed

$$
\begin{aligned}
\operatorname{dim}\left(O_{\hat{\mathfrak{p}}}\right) & =\operatorname{dim}(Z(N) /(Z(N) \cap \hat{P}))=\operatorname{dim}(M /(M \cap \hat{\mathfrak{p}})) \\
& =1 / 2\left[v_{F}(\operatorname{det}(k \mid(M \cap \hat{\mathfrak{p}})))-v_{F}(\operatorname{det}(k \mid M))\right]
\end{aligned}
$$


and

$$
\begin{aligned}
\operatorname{dim}\left(\widetilde{O}_{\hat{\mathfrak{p}}}\right) & =\operatorname{dim}(\widetilde{Z}(N) /(\widetilde{Z}(N) \cap \widetilde{P}))=\operatorname{dim}(\widetilde{M} /(\widetilde{M} \cap(\hat{\mathfrak{p}} \otimes \widetilde{A}))) \\
& =1 / 2\left[v_{\widetilde{F}}(\operatorname{det}(k \mid(\widetilde{M} \cap(\hat{\mathfrak{p}} \otimes \widetilde{A}))))-v_{\widetilde{F}}(\operatorname{det}(k \mid \widetilde{M}))\right],
\end{aligned}
$$

where $v_{\widetilde{F}}$ is the valuation of $\widetilde{F}$. But

$$
\widetilde{M} \cap(\hat{\mathfrak{p}} \otimes \widetilde{A})=(M \otimes \widetilde{F}) \cap \hat{\mathfrak{p}} \otimes \widetilde{A}=(M \cap \hat{\mathfrak{p}}) \otimes \widetilde{A} .
$$

So we see that

$$
\begin{aligned}
v_{\widetilde{F}}(\operatorname{det}(k \mid(\widetilde{M} \cap \hat{\mathfrak{p}} \otimes A)) & =v_{\widetilde{F}}(\operatorname{det}(k \mid(M \cap \hat{\mathfrak{p}}) \otimes \widetilde{A})) \\
& =n v_{F}(\operatorname{det}(k \mid(M \cap \hat{\mathfrak{p}}))) .
\end{aligned}
$$

Besides,

$$
v_{\widetilde{F}}(\operatorname{det}(k \mid \widetilde{M}))=0
$$

because $N$ is split over $\widetilde{F}$, so $\widetilde{M}$ is $G(\widetilde{F})$-conjugate to $\mathfrak{h} \otimes \widetilde{A}$. (Recall that $\mathfrak{h} \subset \mathfrak{g}$ is a Cartan subalgebra.) Substituting (3), (4) in (2) and comparing the result with (1) we get the lemma.

Lemma 3. $v_{F}(\operatorname{det}(k \mid M))=\operatorname{rk}(\mathfrak{g})-\operatorname{dim}\left(\mathfrak{h}^{w}\right)$.

Proof. Since $N$ is split over $\widetilde{F}$, there exists an inner automorphism of $\mathfrak{g} \otimes \widetilde{F}$ which induces an isomorphism

$$
\widetilde{M} \cong \mathfrak{h} \otimes \widetilde{A} .
$$

On the two sides of (5) there is a natural action of the Galois group of $\widetilde{F} / F$. Let $\rho_{l}, \rho_{r}$ denote the corresponding actions. On the RHS we have an action of $W$ (through $\mathfrak{h}$ ), which we denote by $\sigma$. Let $s$ be a generator of $\operatorname{Gal}(\widetilde{F} / F)$. By the definition of $w$ we have $\rho_{l}(s)=\rho_{r}(s) \sigma\left(w^{\prime}\right)$ for some element $w^{\prime} \in W$ conjugate to $w$. (We identified the endomorphisms of the two sides of (5).)

Denote by $q$ the $n$-th primitive root of unity which satisfies the equation $s\left(\varepsilon^{1 / n}\right)=q \varepsilon^{1 / n}$. For any $\lambda \in \mathbb{C}$ let $\mathfrak{h}_{\lambda}$ be the $\lambda$-eigenspace of $w^{\prime}$ acting on $\mathfrak{h}$. Then the $A$-module $M=(\widetilde{M})^{G a l}$ is the direct sum of its submodules

$$
M_{i} \cong \mathfrak{h}_{q^{(n-i)}} \otimes \varepsilon^{(i / n)} A
$$

for $i=0, . ., n-1$. Consider the restriction of the Killing form on $M$. Since the conjugation respects the Killing form, we see that the induced Khuri-Makdisi pairing $M_{i} \times M_{j} \rightarrow A$ is 
nondegenerate $\bmod \varepsilon$

$\varepsilon$ times the one nondegenerate $\bmod \varepsilon$ 0 if $i=j=0$

if $0<i<n, i+j=n$ otherwise.

We see that $v_{F}(\operatorname{det}(k \mid M))=\operatorname{rk}(\mathfrak{g})-\operatorname{dim}\left(\mathfrak{h}^{w^{\prime}}\right)=\operatorname{rk}(\mathfrak{g})-\operatorname{dim}\left(\mathfrak{h}^{w}\right)$. The lemma is proved.

Now we are ready to finish the proof of the Proposition. Applying Lemma 2 to $\hat{\mathfrak{p}} \in O$ we get (using Lemma 3 ):

$$
\operatorname{dim}(\widetilde{O})=n\left[\operatorname{dim}(O)+1 / 2\left(\operatorname{rk}(\mathfrak{g})-\operatorname{dim}\left(\mathfrak{h}^{w}\right)\right)\right],
$$

where $\widetilde{O}$ is the corresponding open orbit of $\widetilde{Z}(N)$ on $\widetilde{X}_{N}$; obviously $\hat{\mathfrak{p}} \otimes \widetilde{A} \in$ $\widetilde{O}$. Since $N$ is split in $\mathfrak{g} \otimes \widetilde{F}$ we know by [KL], $\S 5$ that:

$$
\operatorname{dim}(\widetilde{O})=\operatorname{dim}\left(\widetilde{X}_{N}\right)=\delta_{\widetilde{F}}(N) / 2=n \delta(N) / 2,
$$

where $\delta_{\widetilde{F}}:=v_{\widetilde{F}}\left[\operatorname{det}\left(\operatorname{ad} N: \mathfrak{g} \otimes \widetilde{F} / \mathfrak{z}(N) \otimes_{F} \widetilde{F} \rightarrow \mathfrak{g} \otimes \widetilde{F} / \mathfrak{z}(N) \otimes_{F} \widetilde{F}\right)\right]$. Comparing the last two formulas we get the statement b) of the Proposition.

\section{Acknowledgement}

I express my gratitude to V. Ginzburg for stimulating attention to the work. The paper [KL] was brought to my attention by him. I thank N. Spaltenstein for encouragement in publishing the note.

\section{References}

[K] B. Kostant, Lie group representations on polynomial rings, Amer. J. Math., 85 (1963), 327-404.

[KL] D. Kazhdan and G. Lusztig, Fixed points varieties on affine flag manifolds, Israel J. of Math., 62 (1988), 129-168.

Mathematics Department, Tel-Aviv University, Tel-Aviv, Israel

E-mail address: roman@math.tau.ac.il 